\title{
Licht- und elektronenoptische Untersuchungen an Polystictus versicolor
}

\author{
III. Änderung der Grundplasmastrukturen während der Schnallenbildung*
}

Von M. Girbardt

\begin{abstract}
Aus dem Institut für Mikrobiologie und experimentelle Therapie Jena der Deutschen Akademie der Wissenschaften zu Berlin (Direktor Prof. Dr. med. H. KNöLL)

(Z. Naturforschg. 17 b, 49-53 [1962]; eingegangen am 21. April 1961)
\end{abstract}

\begin{abstract}
Im Verlaufe der Schnallenbildung von Polystictus versicolor ist das Hyaloplasma des Schnallenbereichs gesetzmäßigen Veränderungen unterworfen, die sich lebend in einer Änderung seiner Brechungsindices manifestieren. Das Substrukturbild läßt sich auf Grund der variablen äußeren Morphologie des Schnallenauswuchses den aufeinanderfolgenden Zeitphasen zuordnen.

Das Hyaloplasma mit hohem Brechungsindex ist ausgezeichnet durch vermehrten Gehalt an Ribonucleoproteiden. Im Substrukturbild herrscht in den entsprechenden Zellbereichen die granuläre Komponente vor.

Intraplasmatischer gerichteter Transport der substrukturellen Granula ist wahrscheinlich. Interzelluläre Verlagerung ist nicht oder nur in geringem Maße vorhanden.

Die membranösen Elemente des endoplasmatischen Retikulums sind variabel in Form, Größe und Anzahl. Mit zunehmendem Wassergehalt des Hyaloplasmas kommt es zur Bildung großlumiger Bläschen.

Infolge der weitgehend zugunsten des Kernes verschobenen Kern-Plasma-Relation in der nicht fusionierten Schnalle darf eine intensive Synthese von Zellproteinen angenommen werden. Es wird daher diskutiert, ob der Schnalle die funktionelle Bedeutung eines Plasmareservoirs zukommt.
\end{abstract}

Es ist oft versucht worden, verschiedenen $\mathrm{Zu}$ ständen des Grundplasmas bestimmte Feinstrukturunterschiede zuzuordnen. Im elektronenoptischen Bild sind nach Wohlfarth-Bottermann (1958) ${ }^{1}$, Wohlfarth-Bottermann und Mitarb. (1958 und 1959) ${ }^{2,3}$ Sol-Gel-Umwandlungen des Cytoplasmas eng mit Änderungen der Substruktur korreliert. Im embryonalen Entwicklungsgang ist das endoplasmatische Retikulum [PALADE und Mitarb. $(1954)^{4}{ }^{4}$ sowie die granuläre Grundplasmakomponente $\left[\mathrm{P}_{\mathrm{A}}\right.$ LADE $(1955)^{5}$, Lund und Mitarbb. (1958) ${ }^{6}$ ] starken Veränderungen unterworfen. Durch experimentelle Eingriffe [Hodge und Mitarb. (1956) ${ }^{7}$, Schulz $(1957)^{8}$, Anderson und Mitarb. (1958) ${ }^{9}$ ] geht meist die parallele Orientierung der Membranen verloren, und es entstehen viele unterschiedlich große Bläschen.

Bei Polystictus versicolor besitzt das Hyaloplasma in Längsrichtung der Zelle einen Gradien-

* Teil einer Habilitationschrift der mathematisch-naturwissenschaftlichen Fakultät der Friedrich-Schiller-Universität Jena.

1 K. E. Wohlfarth-Bottermann, Protoplasma 49, 231 [1958].

2 K. E. Wohlfarth-Bottermann u. L. Schneider, Naturwissenschaften 45, 140 [1958].

3 K. E. Wohlfarth-Bottermann u. V. Moericke, Z. Naturforschg. 14 b, 446 [1959].

4 G. E. Palade u. K. R. Porter, J. exp. Medicine 100, 641 [1954].

5 G. E. Palade, J. Biophys. Biochem. Cytol. 1, 59 [1955].

6 H. A. Lund, A. E. Vatter u. J. B. Hanson, J. Biophys. Bio- ten des Wassergehaltes [Girbardt (1960) ${ }^{\mathbf{1 0}}$ ]. Das endoplasmatische Retikulum variiert morphologisch ebenfalls [Girbardt (1961 a) ${ }^{11}$ ]. Es liegt daher die Vermutung nahe, daß der unterschiedliche Wassergehalt des Hyaloplasmas zu verschiedenen Erscheinungsformen des endoplasmatischen Retikulums führt. Gewißheit mußte die Analyse der Schnallenregion bringen. Hier liegen die verschieden hydratisierten Hyaloplasmen, als basaler Pol der einen und apikaler Pol der anderen Zelle, unmittelbar nebeneinander. Der morphologisch nicht verwechselbare Schnallenauswuchs und die Lage der Querwände gestatten eine auch zeitlich weitgehend sichere Zuordnung des elektronenoptischen Bildes zur jeweiligen Entwicklungsphase. Dies gibt die Möglichkeit, aus den „Moment-Aufnahmen“ der elektronenoptischen Bilder in begrenztem Maße auf intraplasmatische Dynamik zu schließen [WoHLFARTH-BottermanN (1959) $\left.{ }^{12}\right]^{*}$.

chem. Cytol. 4, 87 [1958].

7 A. J. Hodge, J. D. McLean u. F. V. Mercer, J. Biophys. Biochem. Cytol. 2, 597 [1956].

8 H. Schulz, Zbl. allg. Pathol. pathol. Anatom. 97, 199 [1957].

9 E. Anderson u. V. L. Breemen, J. Biophys. Biochem. Cytol. 4, 83 [1958].

10 M. Girbardt, Ber. dtsch. bot. Ges. 73, 227 [1960].

11 M. Girbardt, Arch. Mikrobiol. 39, 351 [1961 a].

12 K. E. Wohlfarth-Bottermann, Z. Zellforsch. mikroskop. Anatom. 50, 1 [1959].

* Für wertvolle technische Hilfe habe ich Frau I. Bähring, Frl. E. Fritsche und Herrn M. Beyer zu danken. 


\section{Material und Methode}

Kultur- und Präparationsmethoden sind die gleichen wie in den vorangehenden Arbeiten [GIRBardT (1960 und 1961 a) ${ }^{10,11}$ ]. Der RNS-Nachweis erfolgt mit Hilfe einer der von Einarson (1951) ${ }^{13}$ und SAndRITTER (1952 und 1955) ${ }^{\mathbf{1 4}, 15}$ oder Brachet (1953 und 1954) ${ }^{16,17}$ angegebenen Methoden. Die ObjektträgerAgar-Kulturen werden mit 70-proz. Äthylalkohol bzw. nach Carnoy $2 \mathrm{Stdn}$. fixiert (der hohe Prozentsatz plasmoptysierender Hyphen läßt sich etwas herabsetzen durch Fixierung bei $-30^{\circ}$ [Kohlensäureschnee]). Nach 2stdg. Wässern kommen die Objektträger in RNase-Lösung $\left(0,5 \mathrm{mg} / \mathrm{cm}^{3}\right.$ oder $1 \mathrm{mg} / \mathrm{cm}^{3}$ in dest. Wasser $p_{\mathrm{H}} 6,0$ oder Veronal-Acetat-Puffer $p_{\mathrm{H}}$ 6,5 [0,014-m.]) und verbleiben dort bei $37^{\circ} \mathrm{C}$ 2 Stunden. Kontrollen in dest. Wasser bzw. Puffer laufen stets mit. Nach 1-stdg. Wässern wird mit Gallocyanin-Chromalaun bzw. Methylgrün-Pyronin gefärbt.

\section{Ergebnisse}

Phasenoptische Lebenduntersuchung der Schnallenbildung

Abb. 1 soll eine Vorstellung von den bei der Schnallenbildung ablaufenden Vorgängen vermitteln. Solange der Schnallenauswuchs nicht voll ausgebildet ist (Abb. 1a), liegen die beiden teilungsbereiten Kerne (nur einer ist in der Aufnahme sichtbar) in relativ dunklem Hyaloplasma. Die Zellwände der Hyphe sind nur schwach sichtbar, der Halo-Effekt ist stark ausgeprägt.

2 Min. später (Abb. $1 \mathrm{~b})$ ist der Schnallenauswuchs fertiggestellt, der Zustand des Hyaloplasmas ist praktisch unverändert. In den nächsten 2 Min. (Abb. 1 c) hat der Schnallenauswuchs seine endgültige Gestalt erhalten. Die Kernmembranen sind noch intakt und grenzen den phasenoptisch sehr hell gewordenen Außenkernraum vom lediglich im linken Bildteil bereits veränderten Hyaloplasma scharf ab. Nach weiteren 2 Min. (Abb. 1 d) hat das Hyaloplasma die stark hydratisierte Karyolymphe vollkommen aufgenommen. Dabei wird es aufgehellt, der Halo-Effekt ist verringert und die Zellwände werden deutlich sichtbar. In Abb.le ist, eine Minute später, die Kernteilung gerade abgeschlossen. In dieser Zeit ist keine sichtbare Änderung des Hyaloplasmas eingetreten.

13 L. Einarson, Acta pathol. microbiol. scand. 28, 82 [1951].

14 W. SANDRItTER, Röntgen- u. Laborprax. 6, 18 [1952].

15 W. Sandritter, Z. wiss. Mikroskop. mikroskop. Techn. 62 , 283 [1955].
In der Phase der Querwandbildung (Abb. 1f) wird deutlich, daß in den dazwischenliegenden 11 Min. eine weitere schwache Aufhellung des gesamten Hyaloplasmas im Schnallenbereich unter Bildung kleiner heller Vakuolen stattgefunden hat. Dieser Zustand bleibt fast unverändert auch in den nächsten 9 Min. (Abb.lg) bis unmittelbar vor Fertigstellung der Querwände erhalten. Sobald die Querwand bis auf den submikroskopischen Porus (Girbard $[1958]^{18}$ ) geschlossen ist (Abb. $1 \mathrm{~h}$ ), was in den folgenden 6 Min. geschieht, zeigen die Hyaloplasmen vor und hinter der Querwand Unterschiede. Das vor der Querwand liegende (hier rechts davon) wird allmählich heller. Im Schnallenauswuchs und dem hinter der Wand liegenden $\mathrm{Hy}$ phenbereich, der nunmehr zum apikalen Pol der subterminalen Zelle geworden ist, nimmt die phasenoptische Dichte schwach zu. Der Unterschied verstärkt sich in den nächsten 7 Min. (Abb. li). Bemerkenswert ist, daß noch bei beginnender Fusion (Abb. 1 i) des Schnallenauswuchses das Schnallenplasma etwas dunkler ist als das der subterminalen Zelle. In der Phase des Kernübertritts (Abb. $1 \mathrm{k}$ ) ist dieser Unterschied ausgeglichen, der Schnallenauswuchs ist ein Teil der subterminalen Zelle geworden.

Diese Vorgänge laufen gesetzmäßig bei den meisten Schnallenbildungen ab. Unterschiedlich kann der hyaloplasmatische Wassergehalt der Schnallenregion bei Schnallenbeginn sein. Diese Schwankungen sind jedoch nicht stark (GIRBARDt [1960 ${ }^{10}$ ), da die Schnalle normalerweise im gleichen Zellbereich (Spitzenabstand zwischen 100 und $150 \mu$ ) entsteht und sowohl der absolute Wassergehalt wie der Verlauf des Dichtegradienten von Hyphe zu Hyphe wenig variiert.

In seltenen Fällen, z. B. bei Störung des Wachstums der Spitzenzelle, sind die plasmatischen Dichteverhältnisse umgekehrt, wofür Abb. 2 ein Beleg sein soll. Die Vorgänge laufen bei solchen anomalen Schnallenbildungen zunächst (Abb. $2 \mathrm{a}, \mathrm{b}, \mathrm{c}$ ) normal ab. In der zwischen Abb. $2 \mathrm{~b}$ und d verstrichenen Zeit ist aus nicht näher untersuchten Gründen eine Sistierung des Wachstums der Hyphenspitze eingetreten. Abb. $2 \mathrm{~d}$ zeigt, daß im Gefolge des Wachstumsstillstandes das Hyaloplasma hinter

16 J. Brachet, Quart. J. Micr. Sci., 3. Ser. 94, 1 [1953].

17 J. Brachet, Mikroskopie [Wien] 9, 209 [1954].

18 M. Girbardt, Arch. Mikrobiol. 28, 255 [1958]. 

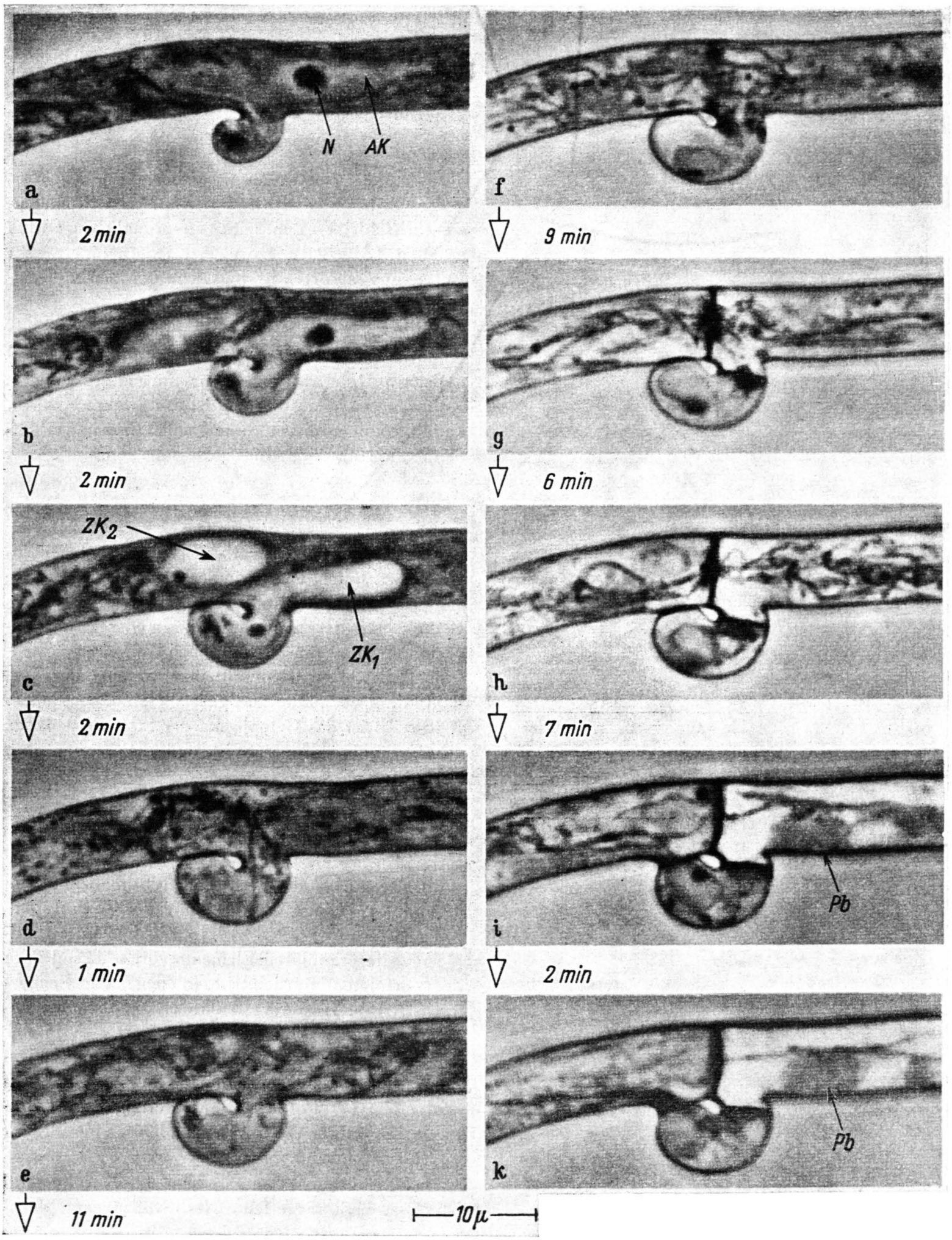

Abb. 1. Polystictus versicolor. Schnallenregion während der Kernteilung und Querwandbildung. Nähere Erläuterungen s. Text. $\mathrm{Ak}=$ Außenkern. $\mathrm{N}=$ Nucleolus. Zk 1 und $2=$ Zellkern 1 und $2 . \mathrm{Pb}=$ "Plasmaballen“, die apikalwärts abwandern. - Phako lebend, $2000: 1$. 

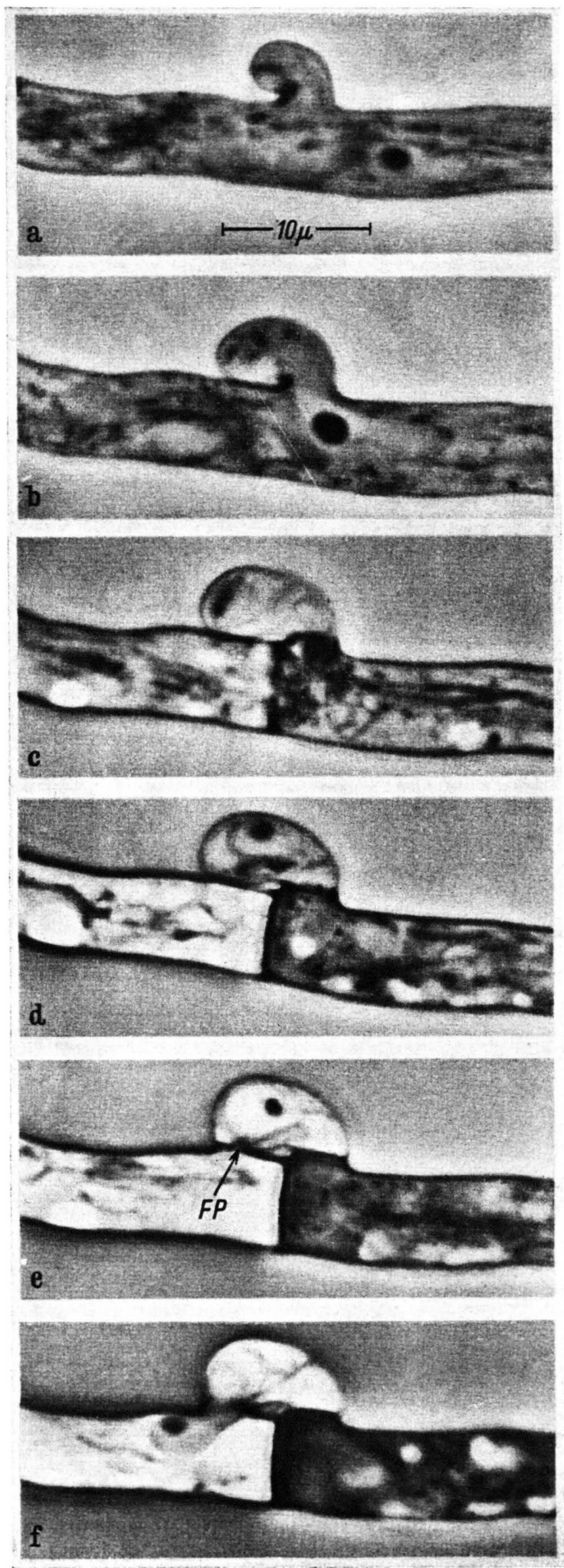

«Abb. 2. Polystictus versicolor. Schnallenregion mit anomaler Hyaloplasma-Hydratation. Im Gegensatz zum Normalfall erfährt der apikale Pol der Subterminalen eine gesteigerte Hydratation. FP = Fusionsporus. - Phako lebend, $2000: 1$.

der Wand - im Gegensatz zu normalen Zellen heller wird. Im zunächst abgeschlossenen Schnallenauswuchs bleibt es in seiner ursprünglichen Dichte erhalten (Abb. 2d). Dort setzt jedoch eine starke Aufhellung sofort ein, nachdem der zentrale Fusionsporus (Abb. 2 e FP) gebildet ist.

Solche Anomalien treten in normal wachsenden Kulturen äußerst selten auf. Da für die elektronenoptischen Untersuchungen nur normal gewachsene Kulturen verwendet werden, ist es unwahrscheinlich, daß Schnallen mit solch anomalen Plasmaverhältnissen in Ultradünnschnitten anzutreffen sind.

$$
\text { Cytochemische Befunde }
$$

Der cytoplasmatische RNS-Gehalt nimmt bei Polystictus versicolor kontinuierlich von der Spitze zur Basis der Zelle ab. Der Basophiliegradient des Cytoplasmas ist gleichlaufend. In der Schnallenregion treffen die Cytoplasmen mit unterschiedlichem RNS-Gehalt unmittelbar aufeinander, sobald die Querwände fertiggestellt sind. Besonders RNS-reich ist das Cytoplasma der Schnalle, solange der Kern eingeschlossen ist und die Fusion noch nicht erfolgte.

\section{Das Substrukturbild der $\mathrm{Schnallenregion}$}

In der Phase des wachsenden Schnallenhöckers (Abb. $3 *$ ) [nähere Beschreibung der Teilungsphasen s. Girbardt $\left(1961\right.$ b) ${ }^{10}$ ] herrschen im Grundplasma die granulären Elemente vor, während nach dem erfolgten Kerneintritt in den Schnallenauswuchs (Abb. 4) die vesikulären Komponenten zunehmen. Nach Abb. $1 \mathrm{c}$ und $\mathrm{d}$ ändert sich in der Phase, der die Abb.4 entspricht, der Brechungsindex des Hyaloplasmas durch Aufnahme der Karyolymphe beträchtlich. Die Änderung des Hydratationsgrades der Grundplasmakolloide spiegelt sich im Substrukturbild.

Das der Abb. $1 \mathrm{~g}$ entsprechende Substrukturbild ist in Abb. 5 dargestellt. Nach vollzogener Kern-

* Abb. 3-7 s. Tafel S. 52 a u. b.

19 M. Girbardt, Exp. Cell. Res. 23, 181 [1961 b]. 

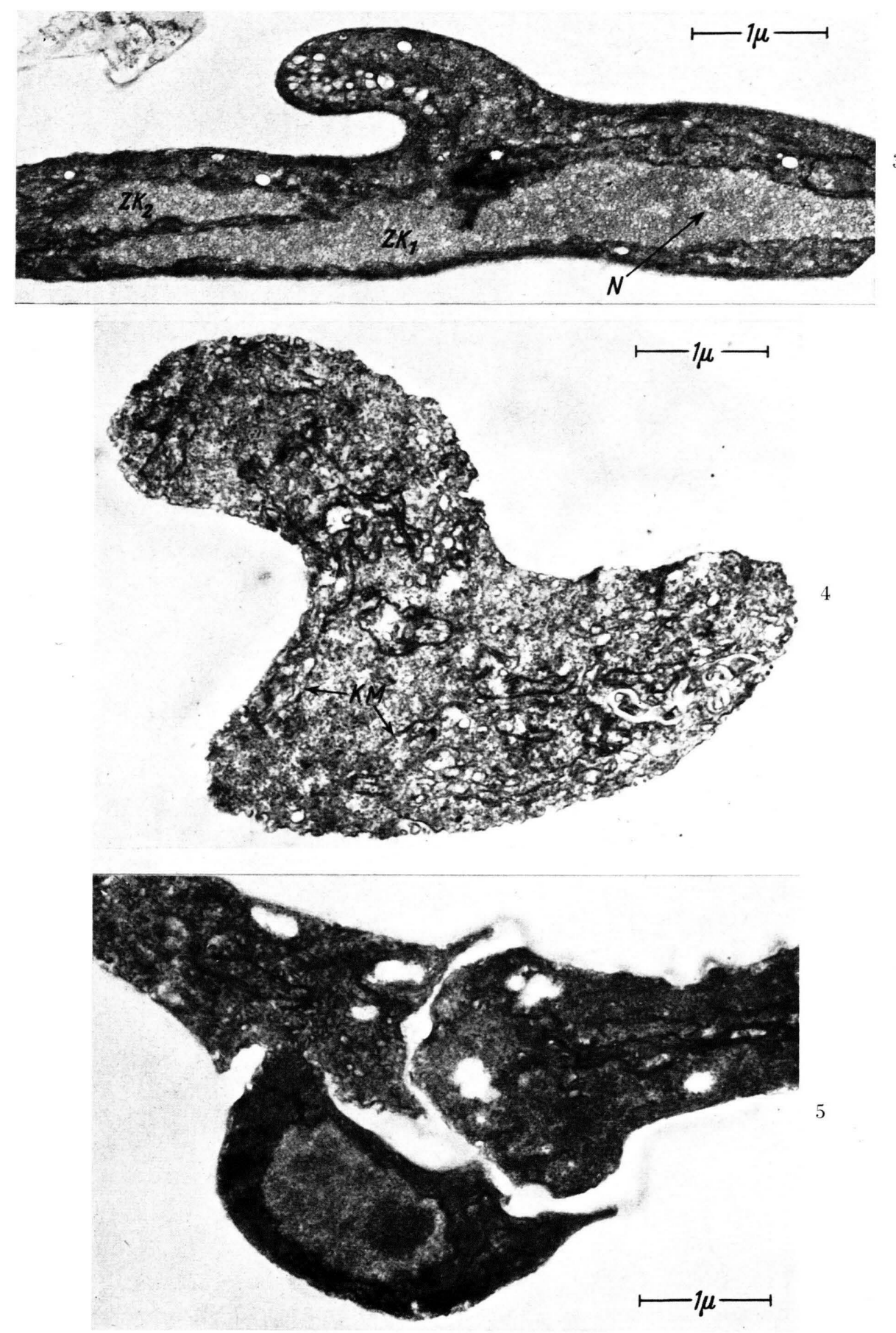

Abb. 3. Polystictus versicolor. Schnallenregion vor Fertigstellung des Schnallenauswuchses. Zk 1 und 2 = Zellkern 1 und 2. - Elmi, $25000: 1$.

Abb. 4. Polystictus versicolor. Schnallenregion in der Phase des sich auflösenden Mutterkernes. $\mathrm{KM}=$ Kernmembran. - Elmi, $25000: 1$.

Abb. 5. Polystictus versicolor. Schnallenregion nach vollzogener Kernteilung mit noch im Schnallenauswuchs eingeschlossenem Tochterkern und beginnender Fusion. - Elmi, 25000 : 1. 


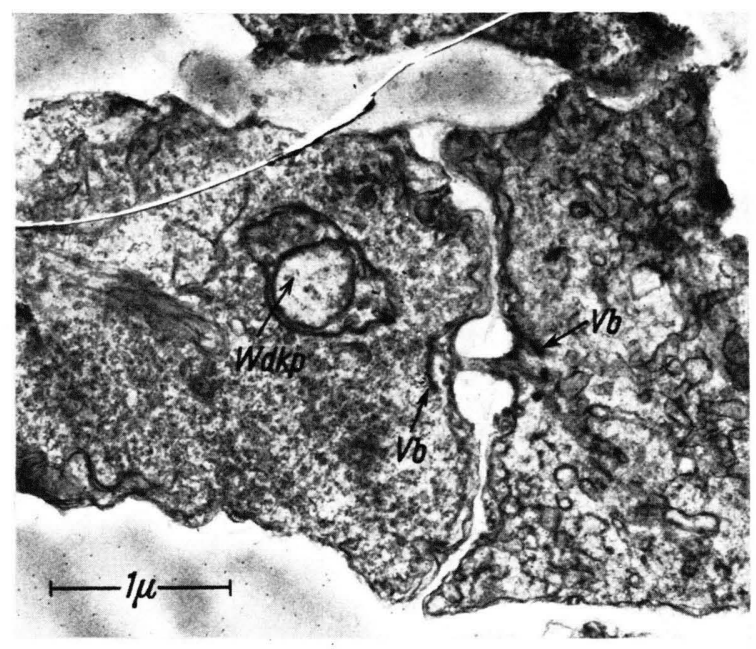

6

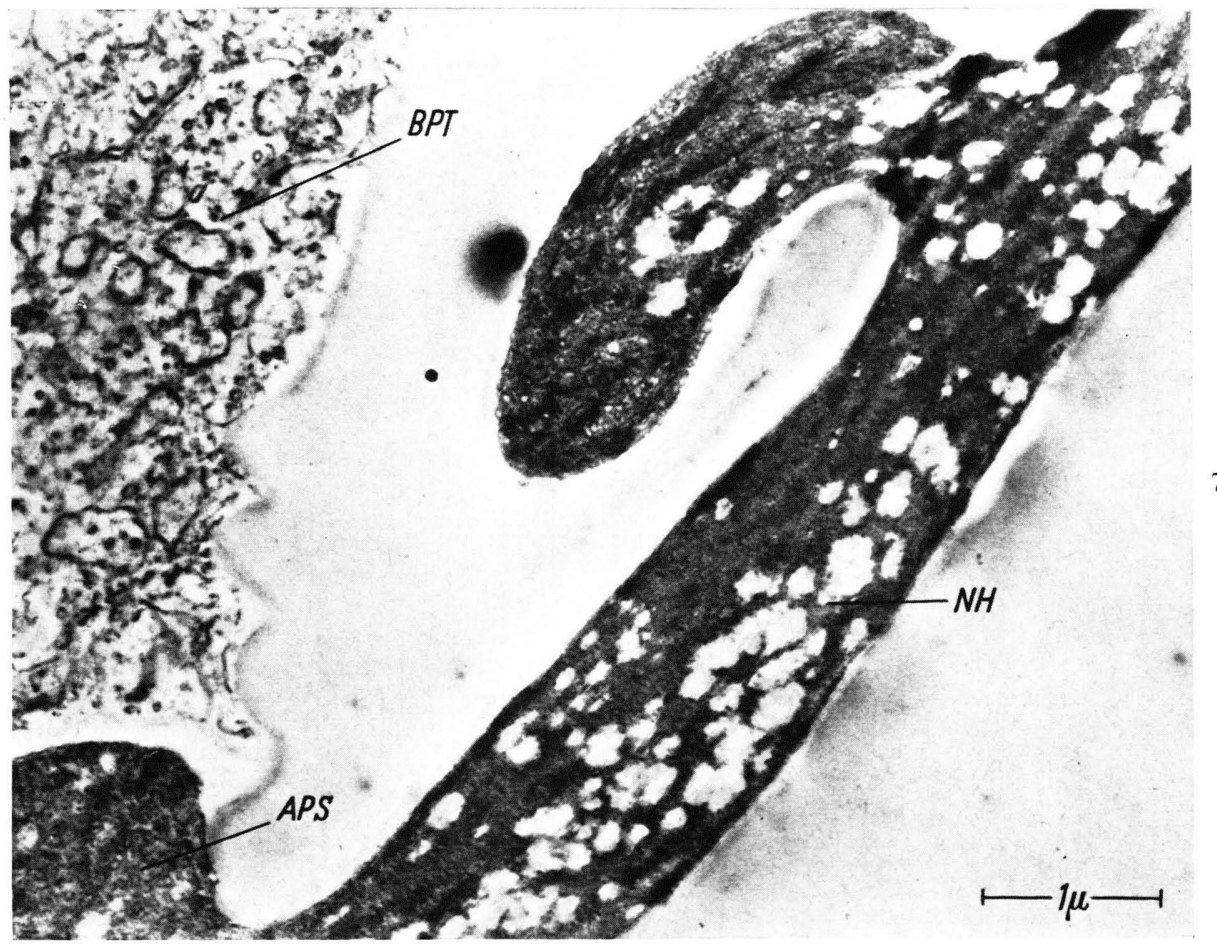

Abb. 6. Polystictus versicolor. Schnallenregion nach erfolgter Fusion des Schnallenauswuchses. Wdkp. = vermutl. „Wandkörper“ chondriosomaler Herkunft. Vb=Verschlußband für Wandporus. - Elmi, $25000: 1$.

Abb. 7. Polystictus versicolor. Basaler Pol der Terminalzelle (BPT) und apikaler Pol der Subterminalzelle (APS) mit ausgewachsener Nebenhyphe (NH). - Elmi, $25000: 1$. 
teilung nimmt die Anzahl der Granula wieder zu. Vor und hinter der Wand sind keine merklichen Unterschiede zu konstatieren. Im Schnallenauswuchs liegen die Granula besonders dicht gepackt. An der späteren Fusionsstelle ist der Unterschied zwischen Schnallen- und Haupthyphenplasma deutlich zu erkennen.

Nach erfolgter Fusion des Schnallenauswuchses (vgl. Abb. $1 \mathrm{~h}-\mathrm{k}$ ) treten die stärksten hyaloplasmatischen Veränderungen im basalen Ende der Terminalzelle auf. Auch dieFeinstrukturen (Abb.6) sind in diesem Zellbereich am meisten verändert. Eindeutig beherrschen die Bläschen des endoplasmatischen Retikulums das Bild. Die Größe der Bläschen steigt mit zunehmender Hydratation, so daß die Unterschiede zwischen den durch eine perforierte Querwand geschiedenen Grundplasmen besonders auffällig werden, wenn die Nebenhyphe bereits ausgewachsen ist und sich zur Schnallenbildung anschickt (Abb. 7).

\section{Besprechung der Ergebnisse}

Dem unterschiedlichen RNS-Gehalt läuft die unterschiedliche Dichte der P a l a d e schen Granula parallel. Ungeachtet der bislang nicht eindeutig beant. wortbaren Frage, ob die Granula (Ribosomen) prämortal existieren oder nur Fällungsprodukte darstellen, sind sie als Träger der RN-Proteide anzusprechen. Ihre Häufung kennzeichnet bei Polystictus versicolor Zellbereiche, in denen Wachstumsprozesse (Nebenhyphenbildung) ablaufen.

Ein interzellulärer Transport der Granula über vorhandene Grundplasmaverbindungen scheint nicht oder nur in geringem Maße stattzufinden, sonst wäre der scharf ausgeprägte Unterschied unmittelbar nebeneinanderliegender Grundplasmen (vgl. Abb.5) nicht verständlich. Innerhalb der Zelle scheinen jedoch gerichtete Verlagerungen möglich zu sein. Die Verringerung der Granulazahl im

20 M. Girbardt, Flora [Jena] 142, 540 [1955].

21 B. Blondel u. G. Turian, J. Biophys. Biochem. Cytol. 7, 127 [1960]. basalen Zellbereich nach Fertigstellung der Querwände kommt wahrscheinlich durch akropetalen Transport der Granula zustande. Hierfür sprechen auch die lebend beobachtbaren, abwandernden „Plasmaballen“ [Girbardt (1955) ${ }^{20}$, vgl. auch Abb. $1 \mathrm{i}, \mathrm{k}$ ]. Blondel und Turian (1960) ${ }^{21}$ halten einen intraplasmatischen Transport der Granula bei Allomyces ebenfalls für möglich.

Weit ungewisser sind die Aussagen über Genese und Formenvariabilität der membranösen Elemente. Die vorliegenden Untersuchungen stellen sicher, daß mit zunehmendem Wassergehalt des Hyaloplasmas die großlumigen Blasen vorherrschen. Diese Blasen brauchen miteinander nicht mehr in Verbindung zu stehen. Die zeitlich aufeinanderfolgenden Erscheinungsformen lassen die Deutung zu, da $\beta$ die Blasen durch Quellungsprozesse aus den englumigen Tubuli entstehen. Diese Prozesse müssen reversibel sein, wie ein Vergleich der Abb. 4 mit Abb. 5 zeigt.

Die Ergebnisse sind auch im Zusammenhang mit einer möglichen funktionellen Bedeutung der Schnallenbildung einer Betrachtung wert. Bei Polystictus versicolor (wie auch bei vielen anderen Hymenomyceten) entsteht die Nebenhyphe mit großer Regelmäßigkeit aus dem apikalen Teil der Subterminalen. Viele Lebendbeobachtungen haben gezeigt, daß die Nebenhyphe häufig erst auswächst, nachdem die Fusion der Schnalle stattgefunden hat. Die phasenoptischen (vgl. Abb. li) sowie die elektronenoptischen (vgl. Abb.5) Bilder zeigen, daß im Schnallenauswuchs sehr „dichtes“ Plasma gebildet werden kann. Es wäre vorstellbar, daß die Schnallen eine Art Reservoir darstellen, in dem auf Grund der günstigen Kern-Plasma-Relation eine intensive Synthese von Proteinen stattfinden kann. Die Schnalle entspräche damit auch funktionell dem Ascomyceten-Haken, dessen wahrscheinlich ursprüngliche Bedeutung [GREIS (1937) ${ }^{22}$ ] darin liegt, die ascogenen Hyphen zu vermehren.

22 H. Greis, Jb. wiss. Bot. 84, 740 [1937]. 